PAPER

\title{
Transferring technical listening training curriculum at the Department of Acoustic Design, Kyushu University, to a corporation in the acoustics industry
}

\author{
Kazuhiko Kawahara ${ }^{1, *}$, Masayuki Takada ${ }^{1}$, Shin-ichiro Iwamiya ${ }^{1}$ and Toshihiro Ito $^{2}$ \\ ${ }^{1}$ Faculty of Design, Kyushu University, \\ Shiobaru 4-9-1, Minami-ku, Fukuoka, 815-8540 Japan \\ ${ }^{2}$ Yamaha Corporation, Nakazawa-cho 10-1, Naka-ku, Hamamatsu, 430-8650 Japan
}

(Received 31 July 2015, Accepted for publication 12 January 2016)

\begin{abstract}
Technical listening training (TLT) is a systematic training program designed to improve auditory sensitivity. TLT consists of discrimination and identification tasks for a wide variety of acoustic features, e.g., frequency, sound pressure level, and spectrum pattern. To improve the auditory sensitivity of employees in a corporation in the acoustics industry, the TLT curriculum at the Department of Acoustic Design, Kyushu University (KU), was transferred to the corporation as part of a corporate education program. An initial 5-day trial course was carried out by the KU staff to demonstrate the process of KU-style TLT and its effectiveness to the corporate staff. After the creation of a TLT control system and training room, a second trial 11-day course was jointly carried out by KU and the corporate staff as a simulation of the TLT course in the corporation. Participants in the trials were engineers in the corporation. Through the TLT trials, they improved their auditory sensitivity and their understanding of the relationship between acoustic properties and auditory attributes. They recognized the importance of the systematic listening experiences provided by TLT. Through these two trials, the corporate staff gained understanding of the TLT curriculum. Therefore, the corporation has begun running the TLT curriculum smoothly and successfully.
\end{abstract}

Keywords: Acoustic education, Corporate education, Auditory sensitivity, Listening experience, Acoustics industry

PACS number: 43.10. Sv [doi:10.1250/ast.37.157]

\section{INTRODUCTION}

The Department of Acoustic Design at Kyushu University (KU) provides an education program for sound experts, such as acoustic engineers and sound designers. Sound experts must have a basic and broad knowledge of acoustics and the latest information on audio technology. In addition, sound experts are required to have special auditory sensitivity, which they acquire through various experiences during their careers.

Technical listening training (TLT) is a systematic training program designed to improve auditory sensitivity $[1,2]$. TLT was developed at the Department of Acoustic Design, Kyushu Institute of Design (later joined to Kyushu University). Through this program, students can obtain the necessary auditory sensitivity before gaining work experience on the job. TLT provides a wide variety of

\footnotetext{
*e-mail: kawahara@design.kyushu-u.ac.jp
}

experiences, so that students can obtain auditory sensitivity associated with various fields and easily adapt to new auditory environments after graduation.

In Japan, companies generally have their own educational programs for employees. Some companies in acoustics industries are interested in introducing TLT as a part of education for acoustic engineers. In the past, acoustic engineers acquired acoustic sensitivity on their own through their work experience. However, according to changes in working style and the tough requirements of job efficiency, they do not have enough opportunities to listen to various kinds of sounds at their job sites currently. In particular, the progress of digital technology has affected the working environment. For example, the computer-aided design process is a powerful and convenient tool for making products to match the acoustic requirements of the product plan. However, in this process, engineers mainly rely on graphic expression of acoustic features and do not have many listening experiences. Crucially, colleagues do not share the 
same listening experiences. Therefore, even mature acoustic engineers do not have the required auditory sensitivity, and they cannot share their sensitivities to sounds with their group. Under these circumstances, a corporation in the acoustics industry was interested in introducing TLT to provide systematic listening experiences for acoustic engineers and allow them to gain the necessary auditory sensitivity. The business activities of the corporation are wide and range into various acoustics industries and related fields, such as musical instruments, audio equipment, room acoustics, music software and music education. In terms of corporate culture, sensitivity to sounds is assumed to be an important aspect for employees of the corporation. However, they also faced the above-mentioned serious problems. A decrease in listening experiences on job sites might cause a serious situation for the succession of corporate culture for sound creation. The corporation was seeking a way of educating employees in auditory sensitivity. TLT seemed to be a solution for the corporation.

There are various types of TLT run in Japanese universities [3-5]. TLT at the Tokyo University of Information Science [3] is an excellent curriculum for general students (not majors in acoustic fields) to acquire sensitivity to sounds, but was not necessarily appropriate to apply to systematic training for acoustic engineers. TLT (Critical Listening) at Tokyo University of the Arts [4] is specialized for recording engineers and is a very intensive and effective curriculum for them, but does not contain enough variety for wider fields of acoustics industries. TLT at the Kanazawa Institute of Technology was not on the official curriculum when the corporation planned TLT education, although a well-organized TLT curriculum has since been introduced [5]. The corporation decided to introduce the TLT program at KU, because it was a wellorganized and systematic educational program and could be applied to a wide variety of acoustics industries. Furthermore, the actual results of TLT at KU were well recognized in the acoustics industries. However, the corporation did not have enough knowledge and experience of this type of curriculum. According to the request of the corporation, teaching staff at the Department of Acoustic Design, KU decided to cooperate to establish a TLT program for acoustic engineers in the corporation. This paper reports on the process of transferring the TLT curriculum at a university to the industry.

There are other cases of introducing TLT in acoustics industries in Japan [6,7]. Recently, in Europe, an example of transferring TLT education from academic organizations to industry was reported [8]. As shown in workshops on ear training at the Audio Engineering Society conventions, concepts and methods for ear training, such as TLT, have become widespread $[9,10]$. This trend might be more general in the future. Some of the authors actually consulted with some companies that have planned their own TLT program. This article might be an informative report for companies that are interested in introducing TLT as corporate education.

\section{BASIC CONCEPT OF TLT AT THE DEPARTMENT OF ACOUSTIC DESIGN, KYUSHU UNIVERSITY, AND ITS APPLICATION TO ACOUSTICS INDUSTRIES}

As a fundamental ability, sound experts should have the ability to discriminate different sounds, and should be able to identify various types of perceived differences, such as pitch, loudness and timbre. As shown in the TLT syllabus at KU, TLT begins with discrimination tasks covering pitch, loudness and timbre.

Furthermore, sound experts should also develop the ability to correlate these auditory differences with the physical properties of sounds. When a sound expert needs to explain an auditory difference, this difference should be expressed using the appropriate technical terms. This ability enables sound experts to imagine the proper sounds when given the acoustic properties of the sounds, just as expert musicians can imagine music by looking at a score. TLT provides various types of identification tasks of acoustic features, such as sound pressure level, frequency, and spectrum pattern.

Through TLT, to become sound experts, students improve their auditory sensitivity and their understanding of the relationship between acoustic properties and auditory attributes. The training tasks of TLT were developed to be similar to the listening experiences of mature acoustic engineers and designers. TLT can provide them various and systematic listening experiences, which is effective to acquire the required auditory sensitivity. The concept of TLT can apply to corporate education in acoustics industries.

\section{PLANNING STAGE}

As a first step, the teaching staff of TLT at KU and the members of the TLT project at the corporation discussed the basic concept of the TLT curriculum in the corporation and planned the process of transferring the understanding of the TLT education method. The corporation wanted to introduce a KU-style TLT curriculum as part of corporate education. Essentially, they thought that TLT contributed to raising the level of the engineers in the corporation. They also thought that TLT was good education for improving the skills of craftspeople of musical instruments. Furthermore, they wanted to extend the very basic stages of TLT to other employees, as they expected that TLT experience contribute to improving the corporate culture of the corporation. However, KU and the corporate staff agreed that the first target on this project was engineers. 
$\mathrm{KU}$ and the corporate staff planned to have two-stage trial courses of TLT in the corporation for the smooth transfer of the TLT curriculum. The first stage trial consisted of a 5-day training course with lectures on fundamental acoustics. The style of this course was similar to that of TLT at KU. During this trial, the teaching staff of KU taught the TLT educational method to the corporate staff, and both sets of staff analyzed the results of TLT, judged its appropriateness for corporate engineers, and discussed how to better optimize the educational process. Before the trial courses began, the corporate staff observed a TLT class at KU and got clear ideas of TLT.

The second stage trial was an 11-day training course, which was assumed to be the TLT curriculum for the corporation. The aim of this trial was a simulation of an actual TLT course in the corporation. After the first stage, the TLT system in the corporation was developed, based on the KU system [11]. The aim of the second stage trial included a trial run of this system.

\section{FIRST STAGE TRIAL: DEMONSTRATION OF TLT TO THE CORPORATION}

The first stage trial was carried out in a meeting room of the corporation. The aim of this trial was to demonstrate the TLT program at KU to the corporate staff and to obtain recognition for this project in the corporation. The corporate staff learned about TLT education and clarified what they should do to run TLT by themselves. This trial was a 5-day training course of 60-minute training sessions.

The style of this course was similar to that of TLT at $\mathrm{KU}$. In addition to the training syllabus, brief lectures on acoustic properties related to the training syllabus were given to participants. The contents of the lectures included frequency and amplitude of pure tones, spectrum and harmonics, sound pressure level, and frequency characteristics of colored music and band noise. The lecturer also explained the aims of each training session.

The teaching staff of KU managed the training progress, including instructing and teaching. The course participants were 12 engineers from the corporation, ranging in age from their $20 \mathrm{~s}$ to $50 \mathrm{~s}$. Most of them were in charge of acoustic engineering or acoustic design. All of the training sessions, including the instructions and lectures, were video recorded. Later, they were analyzed and documented as a TLT manual. The provided training syllabus and schedule are shown in Table 1. This syllabus was the digested version of the basic TLT course at KU, which was provided for freshmen by the university. The presentation of training sounds was controlled by a $\mathrm{KU}$ system installed on a notebook PC. Participants wrote their answers on a response sheet after each sound presentation. After each session, the correct answers were aurally
Table 1 Training syllabus and schedule of the first trial of TLT.

\begin{tabular}{|c|c|c|c|c|c|}
\hline \multirow[b]{2}{*}{ Training } & \multicolumn{5}{|c|}{ Number of days } \\
\hline & 1 & 2 & 3 & 4 & 5 \\
\hline Pitch Discrimination & $\mathrm{x}$ & $\mathrm{x}$ & & & \\
\hline Loudness Discrimination & $\mathrm{x}$ & $\mathrm{x}$ & & & \\
\hline Timbre Discrimination & $\mathrm{x}$ & $\mathrm{x}$ & & & \\
\hline $\begin{array}{l}\text { SPL difference Identification } \\
\qquad(10 \mathrm{~dB} \text { stepwise })\end{array}$ & $\mathrm{x}$ & & & & \\
\hline $\begin{array}{l}\text { SPL difference Identification } \\
\text { (5 dB stepwise })\end{array}$ & & $\mathrm{x}$ & $\mathrm{x}$ & & \\
\hline $\begin{array}{l}\text { SPL difference Identification } \\
\text { (2 } \mathrm{dB} \text { stepwise })\end{array}$ & & & & $\mathrm{x}$ & $\mathrm{x}$ \\
\hline $\begin{array}{c}\text { Identification of the center } \\
\text { frequency of processed music } \\
\text { Lower bands: } 125 \mathrm{~Hz}, 250 \mathrm{~Hz} \text {, } \\
500 \mathrm{~Hz} \text {, and } 1 \mathrm{kHz}\end{array}$ & & $\mathrm{x}$ & $\mathrm{x}$ & & \\
\hline $\begin{array}{c}\text { Identification of the center } \\
\text { frequency of processed music } \\
\text { Higher bands: } 1 \mathrm{kHz}, 2 \mathrm{kHz}, 4 \mathrm{kHz} \text {, } \\
\text { and } 8 \mathrm{kHz}\end{array}$ & & & $\mathrm{x}$ & & \\
\hline $\begin{array}{l}\text { Identification of the center } \\
\text { frequency of processed music } \\
\text { All bands: } 125 \mathrm{~Hz}, 250 \mathrm{~Hz} \\
500 \mathrm{~Hz}, 1 \mathrm{kHz}, 2 \mathrm{kHz}, 4 \mathrm{kHz}, \\
\text { and } 8 \mathrm{kHz}\end{array}$ & & & & $\mathrm{x}$ & $\mathrm{x}$ \\
\hline
\end{tabular}

provided to the participants. The training sounds were presented via loudspeakers.

Similar to TLT at KU, the training began with discrimination tasks for pitch, loudness and timbre to enhance sensitivity to auditory differences. The pitch discrimination task was to indicate whether the comparison tone was "higher or lower" than the standard tone when listing to tone pair, "louder or softer" for loudness, and "same or different" for timbre. Through these discrimination experiences, participants became familiar with careful listening.

After the discrimination tasks, the training shifted to the identification of sound types. At the rehearsal sessions of this type of training, participants learned and memorized the auditory features of various variations of physical properties of sounds while listening to the training sounds. Afterward, the participants performed identification tasks on randomly presented training sounds.

In the identification tasks for the difference of sound pressure level, a short music excerpt was presented twice. The first sound was the standard, and the second sound was attenuated from the standard. At the rehearsal session, participants listened to a series of sound pairs, and correlated the loudness differences to differences in the 
sound pressure level. Next, the participants performed identification tasks for randomly presented sound pairs. The stepwise sound pressure level difference between two sounds was $10 \mathrm{~dB}(0,10,20$ and $30 \mathrm{~dB}), 5 \mathrm{~dB}(0,5,10,15$, and $20 \mathrm{~dB})$, and $2 \mathrm{~dB}(0,2,4,6,8$, and $10 \mathrm{~dB})$. The smaller the step, the more difficult the difference was to identify. Through this training, participants acquired the perceptual frame of the loudness difference in terms of decibels.

In the identification of the center frequency of the enhanced octave-bandwidth of the processed sound in recorded music, participants listened to a pair of a short music excerpts: one was the standard and the other was the characterization of its acoustic spectrum. In this training task, a one-octave frequency region of the standard sound was boosted by $10 \mathrm{~dB}$. The participants' task was to identify the center frequency of boosted octave-bandwidth. The center frequency of the enhanced bandwidth was 125 , $250,500,1,000,2,000,4,000$ or $8,000 \mathrm{~Hz}$. Through this training, participants learned the auditory characteristics of each frequency region of musical sounds and gained an understanding of the spectrum patterns of the reproduced sounds.

The other training task involving frequency was the identification of pure tone frequencies and the center frequency of band noises. The frequency of these training tasks was also an octave-band step: 63 (only for band noise), 125, 250, 500, 1,000, 2,000, 4,000 and 8,000 Hz. This training helps participants to gain a sense of frequency and spectrum. Furthermore, the identification tasks involved a number of harmonic components to help to provide a sense of the spectrum and harmonics.

Analysis of the responses by participants showed that their achievement level was equivalent to the level of students at KU. The participants developed the ability to correlate the auditory differences with the physical properties of sounds through TLT. After this course, positive comments on TLT were obtained from the participants. All of the participants admitted the value of TLT in terms of corporate education. They also admitted the importance of the lectures related to the training syllabus. They realized the importance of obtaining systematic auditory memory on the relationship between the perception of sound and the acoustical properties of sounds. Furthermore, they indicated the importance of sharing systematical listening experiences in the group.

The corporate staff realized the effectiveness of KUstyle TLT from the observations of the training course, the achievement level following training, and the feedback from the participants. They came to understand TLT through observing the training sessions, and confirmed that KU-style TLT could be applied to corporate TLT without major modification. In addition, the corporate staff suggested new training tasks for TLT, which were closely related to jobs in acoustics industries and seemed to be effective in maintaining the motivation of participants. We decided to carry out the second stage trial using a similar process to that of the first stage trial, and proposed a new syllabus for the second trial.

\section{SECOND STAGE TRIAL: SIMULATION OF TLT CURRICULUM IN THE CORPORATION}

The second stage trial was an 11-day training course of 90-minute TLT sessions conducted in a sound proofed training room of the corporation. The 90-minute session was equivalent to a TLT session at KU, and the room was renovated specifically for the TLT program. Similar to the $\mathrm{KU}$ environment, next to the training room, a control room was created. In this stage, the TLT system was equipped for effective training. The system consisted of a host computer, Personal Digital Assistnant (PDA) terminals, and sound-reproducing equipment. The terminals communicated with the host via wireless Local Area Network (LAN). The host computer was set in the control room. After the first stage trial, the TLT system for the corporation was developed. The aim of this trial was to simulate the TLT curriculum in the corporation. During the trial, the TLT system was checked from various points before the corporate TLT started. As shown in Table 2, in addition to the first stage syllabus, some training tasks were newly added: the identification of an enhanced frequency band of train noise (the boost frequency was $250,1 \mathrm{kHz}$, and $4 \mathrm{kHz}$ ), identification of reverberation time of impulsive sound and music (the reverberation time was $0.7,1.4,2.1,2.8$, and $3.5 \mathrm{~s}$ ), and the mixing level balance between vocal and accompanying part (to the standard balance between vocal and accompanying part, the vocal level changed to $+4,+2,0,-2$, and $-4 \mathrm{~dB}$ compared with the vocal level of standard mixing). These training tasks were more practical for acoustic engineers at the corporation, and were supposed to attract the participants' interest.

Successively to the first stage trial, the teaching staff of KU managed the training progress, including the instructing and teaching. The corporate staff operated the training system with the aid of the teaching staff from KU. The presentation of training sounds was controlled by the above-mentioned TLT system equipped in the corporation. This system collected the responses of participants and provided feedback to the participants via PDA terminals. The participants were informed of the correctness of their answer and the correct category, just after their input, and the correct ratio of each session was displayed on the screen of each terminal after each TLT session was completed. Sounds were presented via loudspeakers. The participants of this course were 10 young engineers from the corporation aged from their 20 s to early 30 s who were 
Table 2 Training syllabus and schedule of the second trial of TLT, and the proposed target values of correct ratio and standard training numbers for each training type.

\begin{tabular}{|c|c|c|c|c|c|c|c|c|c|c|c|c|c|}
\hline \multicolumn{14}{|c|}{ Number of days } \\
\hline Training & 1 & 2 & 3 & 4 & 5 & 6 & 7 & 8 & 9 & 10 & 11 & Target & Numbers \\
\hline Pitch discrimination & $\mathrm{x}$ & $\mathrm{x}$ & & & & & & & & & & $90 \%$ & 2 \\
\hline Loudness discrimination & $\mathrm{x}$ & $\mathrm{x}$ & & & & & & & & & & $85 \%$ & 3 \\
\hline Timbre discrimination & $\mathrm{x}$ & $\mathrm{x}$ & & & & & & & & & & $80 \%$ & 3 \\
\hline $\begin{array}{l}\text { SPL difference Identification } \\
\text { (10 dB stepwise) }\end{array}$ & $\mathrm{x}$ & & & & & & & & & & & $90 \%$ & 2 \\
\hline $\begin{array}{l}\text { SPL difference Identification } \\
\qquad(5 \mathrm{~dB} \text { stepwise })\end{array}$ & & $\mathrm{x}$ & $\mathrm{x}$ & $\mathrm{x}$ & $\mathrm{x}$ & & & & & & & $90 \%$ & 4 \\
\hline $\begin{array}{l}\text { SPL difference Identification } \\
\qquad(2 \mathrm{~dB} \text { stepwise })\end{array}$ & & & & & & & $\mathrm{X}$ & $\mathrm{x}$ & & & $\mathrm{x}$ & $75 \%$ & 6 \\
\hline $\begin{array}{l}\text { Identification of the center } \\
\text { frequency of processed music } \\
\text { (Lower bands) }\end{array}$ & & $\mathrm{x}$ & $\mathrm{x}$ & $\mathrm{x}$ & & & $\mathrm{X}$ & & & & & $90 \%$ & 4 \\
\hline $\begin{array}{l}\text { Identification of the center } \\
\text { frequency of processed music } \\
\text { (Higher bands) }\end{array}$ & & & $\mathrm{x}$ & $\mathrm{x}$ & & & $\mathrm{x}$ & & & & & $85 \%$ & 4 \\
\hline $\begin{array}{l}\text { Identification of the center } \\
\text { frequency of processed music } \\
\text { (all bands) }\end{array}$ & & & & & $\mathrm{x}$ & $\mathrm{x}$ & & $\mathrm{x}$ & $\mathrm{x}$ & $\mathrm{x}$ & $\mathrm{x}$ & $80 \%$ & 6 \\
\hline $\begin{array}{c}\text { Identification of } \\
\text { pure tone frequency }\end{array}$ & & & $\mathrm{x}$ & $\mathrm{x}$ & $\mathrm{x}$ & & & & & & & $90 \%$ & 6 \\
\hline $\begin{array}{l}\text { Identification of } \\
\text { the center frequency } \\
\text { of band noise }\end{array}$ & & & & $\mathrm{x}$ & $\mathrm{x}$ & $\mathrm{x}$ & & & & & & $90 \%$ & 6 \\
\hline $\begin{array}{l}\text { Identification of } \\
\text { the number of harmonics }\end{array}$ & & & & & $\mathrm{x}$ & $\mathrm{x}$ & $\mathrm{X}$ & & & & & $70 \%$ & 8 \\
\hline Mixing level balance & & & & & & & $\mathrm{x}$ & $\mathrm{x}$ & $\mathrm{x}$ & $\mathrm{x}$ & & $80 \%$ & 8 \\
\hline $\begin{array}{l}\text { Identification of the center } \\
\text { frequency of processed } \\
\text { train noise }\end{array}$ & & & & & & & & $\mathrm{x}$ & $\mathrm{x}$ & $\mathrm{x}$ & & $85 \%$ & 6 \\
\hline $\begin{array}{l}\text { Identification of } \\
\text { reverberation time } \\
\text { (impulsive sound) }\end{array}$ & & & & & & & & & $\mathrm{X}$ & $\mathrm{x}$ & $\mathrm{x}$ & $80 \%$ & 6 \\
\hline $\begin{array}{l}\text { Identification of } \\
\text { reverberation time } \\
\text { (music) }\end{array}$ & & & & & & & & & & $\mathrm{X}$ & $\mathrm{X}$ & $70 \%$ & 8 \\
\hline
\end{tabular}

in charge of acoustic engineering or acoustic design. Their time in the corporation was between 1 and 4 years. All the participants had experience in playing music of various genres for around 2 to 20 years, but none of them were trained in a similar TLT.

The correct ratios for each session of the representative training tasks are shown in Figs. 1 to 4 . Figure 1 shows the results of the identification of the center frequency of $1 / 3$ octave band noise. The correct ratio improved during training sessions and reached around $90 \%$. Furthermore, the standard deviation was the smallest in the last training session; individual differences among the participants became smaller during training sessions. Figure 2 shows the results for the identification of sound pressure level difference of $5 \mathrm{~dB}$ stepwise. Similar to Fig. 1, the correct ratio improved during training sessions and reached around 90\%. Furthermore, also similar to Fig. 1, the standard deviation became smaller during training sessions.

Figure 3 shows the results of the identification of the enhanced bandwidth of colored music in the range from 125 to $1,000 \mathrm{~Hz}$. The correct ratio improved during training sessions and reached around $90 \%$, and the standard 


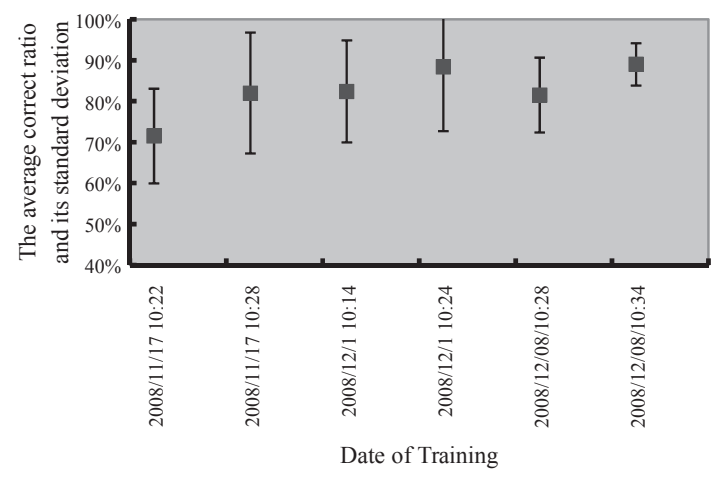

Fig. 1 Results of identification task of center frequency of $1 / 3$ octave band noise: the average correct ratio and its standard deviation for each session.

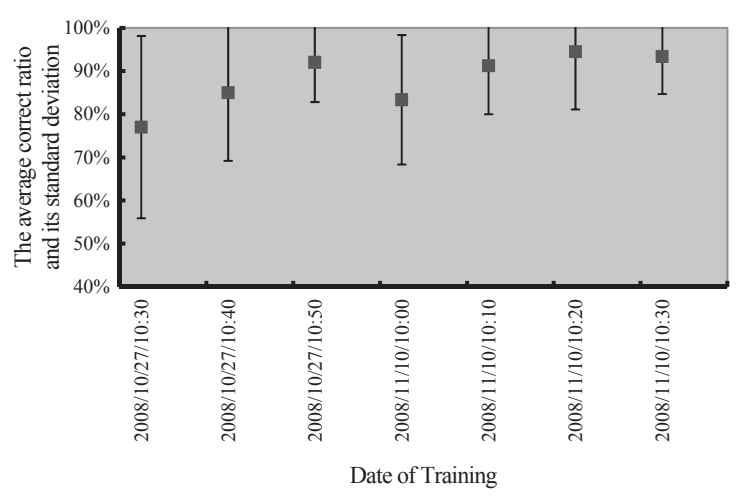

Fig. 2 Results of identification task of sound pressure level difference of $5 \mathrm{~dB}$ stepwise: the average correct ratio and its standard deviation for each session.

deviation became smaller during training sessions. However, for this training type, both the correct ratio and the standard deviation reached to their best values by the fourth training session. Thus, further training sessions might not be necessary for this type of training. Figure 4 shows the results of the identification of the enhanced bandwidth of colored music in the range from 125 to $8,000 \mathrm{~Hz}$. The correct ratio improved during training sessions and reached around $80 \%$, and the standard deviation became smaller during training sessions. Both the correct ratio and the standard deviation reached their best values by the fourth training session. Afterward, the correct ratio fluctuated. During training, sometimes the correct ratio decreased because of a lack of concentration, but this happened sporadically without a clear systematic cause.

After analysis of the correct ratio curves for each training syllabus, the target values of correct ratio and standard training numbers for each training type were proposed as shown in Table 2. Standard training numbers mean the recommended times of training for each training task, which are estimated from the saturation curve of the correct rate. These values are temporary, but clearly

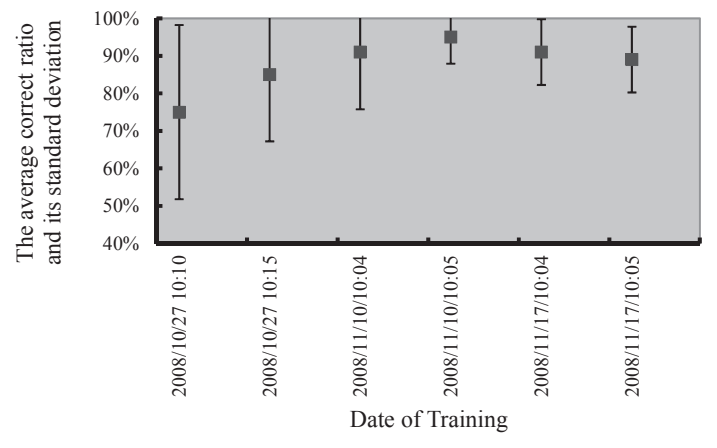

Fig. 3 Results of the identification task of enhanced bandwidth of colored music in the range from 125 to $1,000 \mathrm{~Hz}$ : the average correct ratio and its standard deviation for each session.

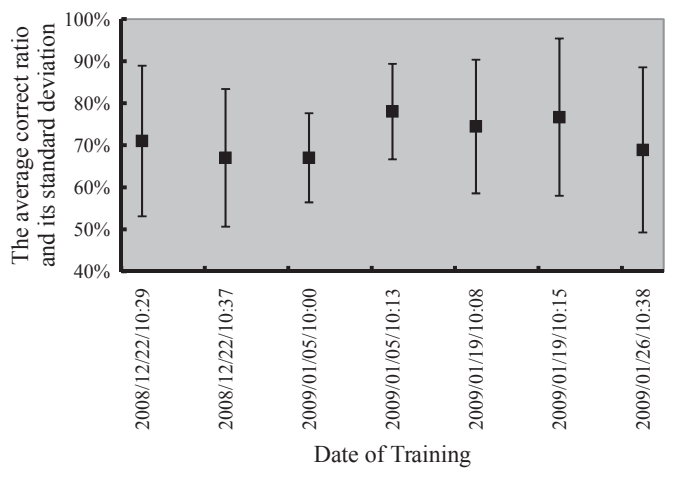

Fig. 4 Results of the identification task of enhanced bandwidth of colored music in the range from 125 to $8,000 \mathrm{~Hz}$ : the average correct ratio and its standard deviation for each session.

proposed targets might be an effective way to maintain participants' motivation.

After this course, the questionnaire survey was conducted. On the question of the effectiveness of TLT for jobs in the corporation, all 10 participants agreed on the effectiveness of TLT. On the question of the systematic auditory memory of the relationship between the perception of sound and the acoustical properties of sounds, all participants said that they had obtained such memory during TLT, and recognized the importance of systematic listening experiences. Furthermore, eight of them commented that their attitude to listening to sounds had changed through TLT. On the question of the appropriateness of the training period, six thought an 11-day training was appropriate, while three thought that it was too short and one thought that it was too long. On the question of training duration, eight thought that a 90-minute training session was appropriate, but two thought that it was too long. On the question of the syllabus of TLT, all thought the provided syllabus for this trial was appropriate. On the question of TLT method, seven approved of the appropriateness of the current TLT method, but three did not and 
preferred a self-training method. On the question of whether they would want to participate in an advanced TLT course, nine wanted to participate. As obtained at the first stage trial, the participants admitted the value of TLT for corporate education.

Throughout the second stage trial, the corporate staff confirmed the effectiveness of TLT. Furthermore, they became familiar with the operation of the TLT system and the process of TLT. The TLT system worked very well, and the staff acquired management ability of TLT. Thus, they completed their preparation for running actual TLT in the corporation.

\section{CURRENT TLT CURRICULUM IN THE CORPORATION}

Currently, the corporation has established three types of TLT programs. The corporate staff manages the TLT curriculum by themselves.

The basic TLT course is provided for newly employed engineers as a part of initiation education. It consists of 5- to 9-day (mainly 9-day) courses of 60- to 120-minute (mainly 90-minute) TLT sessions; the schedule depends on the initiation education schedule each year. The training syllabus is a short-term digested version and consists of basic and applied discrimination tasks: difference of sound pressure level of $5 \mathrm{~dB}$ and $2 \mathrm{~dB}$ stepwise, the center frequency of boost bandwidth of colored music, mixing balance between vocal and accompanying part, and reverberation time. Regardless of their background and expected division in which they will work, all employees of engineering fields participate in this program when they join the corporation. In Japan, newly employed workers generally start their new career in April, and companies provide a corporate education program for them in this season. The basic TLT program is part of such an education program. Freshmen are expected to acquire sensitivity to sounds required as a member of the acoustics industrial company. Increasing the level of auditory sensitivity of employees is expected to bring positive effects on corporate culture in the acoustic industrial company.

The advanced course of TLT is provided for engineers in their thirties: the target is mainly acoustic engineers, but software engineers also participate. The aim of this TLT is to increase the engineers' ability. It consists of 14 days of 90-minute training sessions consisting of basic training and practical training. The training syllabus is similar to that of the second stage trial. Up to now, 21 terms have been completed with about 20 engineers participating in each term. A term means a period over which each participant group completed the TLT syllabus.

After each term, the questionnaire survey was conducted. Figure 5 shows the rates of response categories of "strongly disagree, disagree, neither agree nor disagree,
TLT is as effective as I expected.
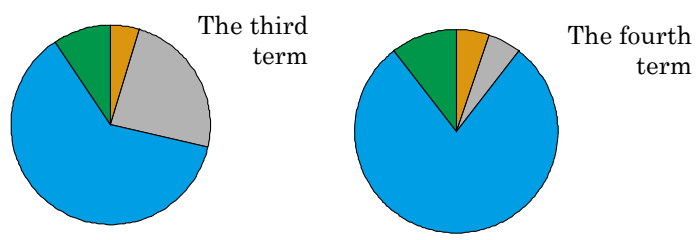

Attitude to listen to sounds or thought to sounds has changed after participated in TLT.
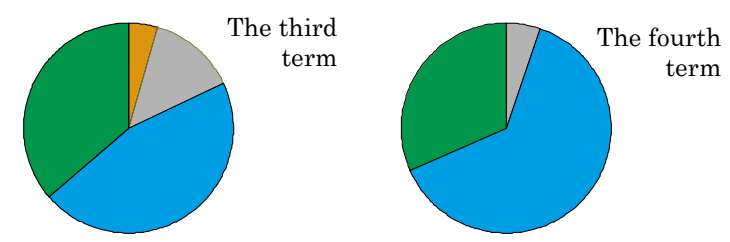

I want to participate in higher-grade TLT.
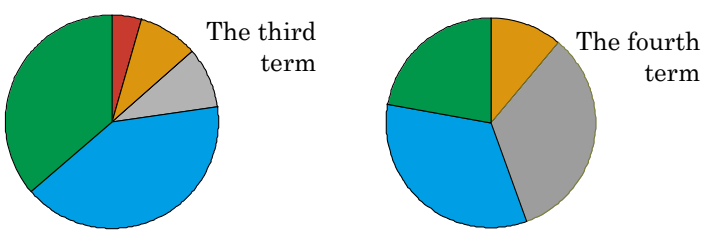

strongly agree

agree

disagree

strongly disagree

neither agree nor disagree

Fig. 5 Results of the questionnaire survey on corporate TLT: rates of response categories for each question. The number of the respondents was 22 for the third term and 19 for the fourth term.

agree, strongly agree" of the participants of the third and fourth term to the questions "TLT is as effective as I expected, attitude to listening to sounds or thinking about sounds has changed after I participated in TLT, I want to participate in higher-grade TLT." The majority of the participants chose the "strongly agree" or "agree" categories of these questions. Many participants admitted the effectiveness of TLT and wanted to continue with further training, although the effect of positivity bias should be taken into consideration in this kind of survey. Furthermore, the participants gave various requests as follows: "I want to learn tips for better discrimination and identification," "I want to do TLT directly related to my job," "want to know how each type of training is effective for different kinds of jobs," "I want to do TLT using wider varieties of sounds and music," and "I want to continue learning TLT using the personal TLT system." These requirements seemed to reflect a positive attitude to TLT. There were also negative comments, such as "I cannot recognize the effectiveness of TLT," and "TLT does not seem to be effective training for acquiring the criterion of goodness of 
sound quality." All these comments gave us hints on how to improve TLT in the corporation.

According to the requirements of a personal TLT system, a web-based TLT system was developed in the corporation. Although this system is at a trial level, employees of the corporation can access this system at any time from anywhere. However, the number of employees who access this system is small. It may not be easy for employees to find training time in their ongoing job situations. Furthermore, because training seems like playing to other employees, they hesitate to join training sessions during work time. Additionally, it may be difficult to maintain motivation at a high level in case of personal training.

\section{CONCLUSION}

The present paper reported the process of transferring the TLT curriculum at the Department of Acoustic Design, Kyushu University (KU), to a corporation. Because KU and the corporate staff sufficiently discussed practical ideas about TLT in the corporation from the planning stage and two stage trials were carried out before starting TLT in the corporation, transferring the TLT curriculum from KU to the corporation was smooth.

Participants in the trials were satisfied with TLT. They developed the ability to correlate the auditory differences with the physical properties of sounds through TLT, and acknowledged its effectiveness. They recognized the importance of systematic listening experiences. Some of them requested advanced courses of TLT. Furthermore, they indicated the importance of sharing systematic listening experiences in the group. Participation in TLT was expected to improve mutual communication about sound quality within the group.

The two stage trials of TLT in the corporation were meaningful for the corporate staff to learn the management of KU-style TLT. The simulation of an actual program was important for the smooth beginning of TLT training. The corporation has begun a TLT curriculum as corporate education and has been running TLT successfully.

\section{ACKNOWLEDGMENTS}

The authors thank Koji Niimi and Tetsu Kobayashi for their support in bringing the TLT projects to the Yamaha corporation.

\section{REFERENCES}

[1] S. Iwamiya, Y. Nakajima, K. Ueda, K. Kawahara and M. Takada, "Technical listening training: Improvement of sound sensitivity for acoustic engineers and sound designers," Acoust. Sci. \& Tech., 24, 27-31 (2003).

[2] S. Iwamiya, "Technical listening training: Training to improve sensitivity to sounds," J. Acoust. Soc. Jpn. (J), 69, 197-203 (2013) (in Japanese).

[3] A. Nishimura, "Significance and outcomes of technical listening training for non-technological university students," Tech. Rep. Acoust. Educ. Acoust. Soc. Jpn., EDU2011-1, pp. 13-18 (2011) (in Japanese).

[4] A. Marui and T. Kamekawa, "A timbral ear training method in musical creativity and the environment of Tokyo University of the Arts," Tech. Rep. Acoust. Educ. Acoust. Soc. Jpn., EDU2011-1, pp. 7-12 (2011) (in Japanese).

[5] N. Emura, H. Adachi, M. Sakurai and M. Yamada, "Training curriculum for technical seeing and hearing," Proc. Autumn Meet. Acoust. Soc. Jpn., pp. 1459-1462 (2014) (in Japanese).

[6] Y. Fukuda, K. Yamakami, A. Koga, K. Miura and T. Kaneko, "Technical listening training for in-house education," Proc. Spring Meet. Acoust. Soc. Jpn., pp. 1449-1450 (2014) (in Japanese).

[7] T. Owaki and M. Tamamura, "Technical listening training for car-audio engineers in company," Proc. Spring Meet. Acoust. Soc. Jpn., pp. 1451-1452 (2014) (in Japanese).

[8] A. Miśkiewicz and T. Letowski, "Timbre solfege training in automotive industry," Proc. Forum Acusticum 2014, 5 pages (2014).

[9] S. Kim, J. Corey, K. Kawahara, A. Marui and S. Olive, "Workshop: Listen professionally or train your ear!," AES 131st Convention New York, 2011 (2011) Available at $<\mathrm{http} / / /$ www.aes.org/events/131/workshops/?ID=2890> (accessed 2015-07-29).

[10] S. Kim, K. Kawahara, D. Ko, S. Vase Legarth, A. Marui, M. McKinnon-Bassett and S. Olive, "Workshop: Listen professionally or train your ear!," AES 132nd Convention Budapest, 2012 (2012) Available at <http://www.aes.org/events/132/ workshops/?ID =3073> (accessed 2015-07-29).

[11] K. Kawahara, M. Takada and S. Iwamiya, "Customized system for technical listening training at Department of Acoustic Design, Kyushu University," J. Acoust. Soc. Jpn. (J), 71, 599600 (2015) (in Japanese). 